\title{
SURGICAL ASPECTS OF GASTRIC AND DUODENAL ULCERATION (EXCLUDING COMPLICATIONS)
}

Twenty to thirty years ago many physicians and surgeons found little justification for surgery in dealing with uncomplicated gastric or duodenal ulcer. Since then the tide has changed and it has become apparent that surgery offers more than medicine, at any rate for the really chronic recurrent ulcer. When the merits of medicine and surgery appeared to be equally balanced, not unnaturally, medical treatment remained the chief standby. After all, to decide on an operation, then of high mortality, required a serious and responsible decision by the surgeon and a rather alarming and difficult step by the patient, in a disease the symptoms of which tended to come and go capriciously. Furthermore, to a surgeon, a surgical mortality is usually a shock, attended by depression and heartsearching, whereas the same man will take a more philosophical and detached view of the death of an ulcer patient from an ulcer complication occurring during medical treatment, even though it might have been avoided by timely operative intervention.

More recent opinion has turned towards surgery because while medical management is efficient in dealing with relapses as they arise, it has made little progress in the prevention of these relapses, except by enforcing a state of invalidism on the patient. Surgery on the other hand has become steadily safer and more efficient, particularly in the last decade.

\section{Selection for Surgery}

Many considerations enter into the question of selection for surgery, apart from the added problems which arise whenever ulcer complications occur. The need for surgery varies directly with the efficiency of the surgical procedure available, and inversely with the operative mortality and morbidity, the chances of recurrent ulceration or of undesirable 'side effects' of the operation. Happily we can now affirm that the patient with chronic gastric or duodenal ulcer can be offered an efficient operation with a very low operative mortality and morbidity rate and with but slight chances of being seriously incommoded by un- $\vec{N}$ pleasant side effects. Let us consider these points in more detail.

The efficiency of the operation. By this we refer particularly to the efficiency of the operation in the prevention of further ulcer symptoms and ulcer complications. In this connection it is important to consider gastric and duodenal ulcer separatel 0 for while the latter is probably a disease resulting from prolonged and abnormal contact of the duodenum with highly acid digestive juice, with possibly a local mucosal lesion as a minor contributory factor, gastric ulceration is frequently and primarily associated with a diseased or degenerative gastric mucosa, and the digestive activity of the gastric juice is less important and possibly only a secondary factor. ${ }^{1}$

Surgical treatment for gastric ulceration means only one thing-partial gastrectomy. No other operation can compare with this in its simplicity and efficiency. The stomal or recurrent gastric ulcer rate after gastrectomy for simple gastric ulcer is exceedingly small. I have been searching for a proven case of this nature for many years and neither among my own cases nor among the many cases sent to my clinic for advice or for gastroscopy $\frac{T}{O}$ from other hospitals was I able to find one until this year. Out of some 698 personal gastrectomies $N$ for gastric ulcer only one patient, a male aged 48 , N developed a stomal ulcer. His original gastric $\mathrm{N}$ ulcer was a remarkable one in that it occurred in $\omega$ the middle of the greater curve of the pyloric antrum (Fig. I), and although it healed satis- $O$ factorily on medical treatment its site was so suggestive of malignancy that a two-thirds partial gastrectomy was carried out. The stomal ulcer, which was diagnosed one year later, was dealt with by the addition of a vagotomy. 


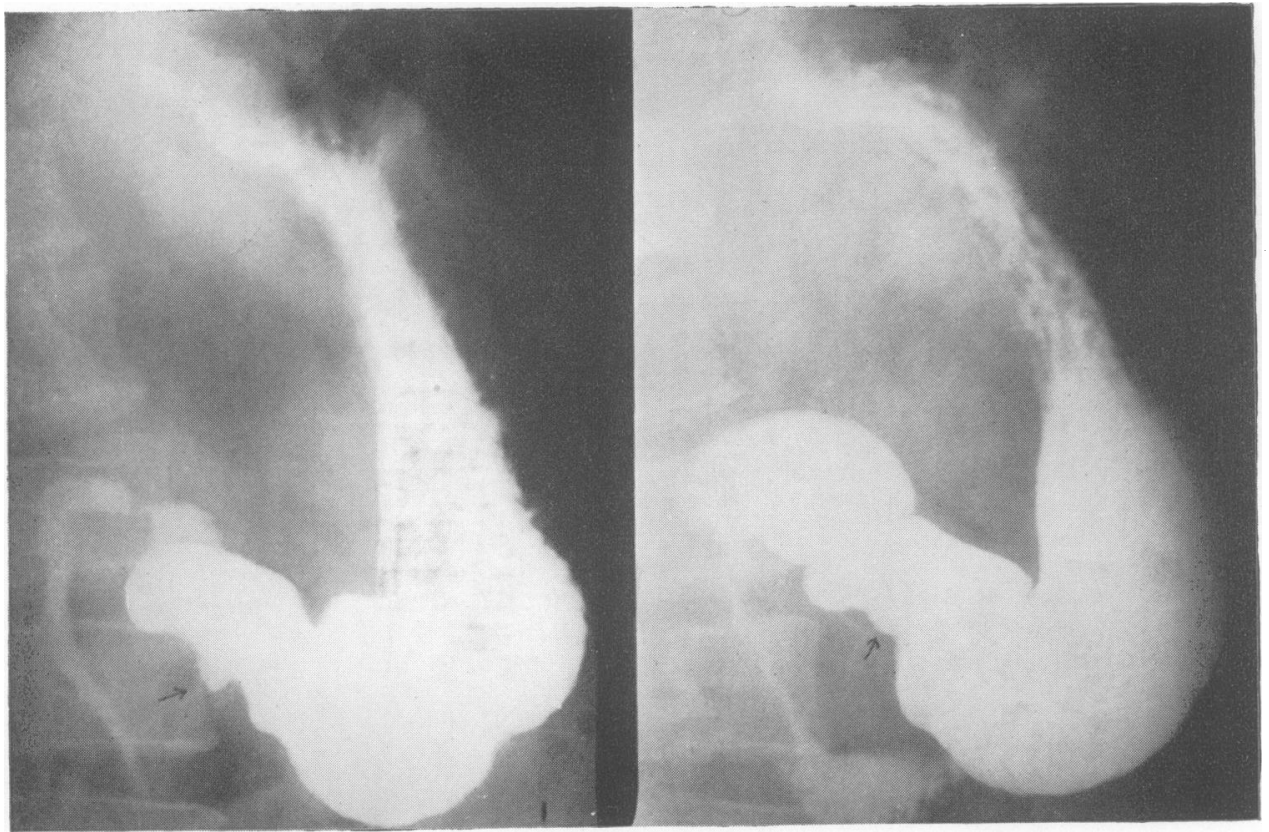

FIG. I.-X-ray of a simple pre-pyloric greater curvature gastric ulcer before and after medical treatment. This is the only case of the writer in which stomal ulceration has followed gastrectomy for gastric ulcer.

There is no doubt that whenever functioning stomach and small bowel are anastomosed there is always the possibility of stomal ulceration. One can state, however, that such an anastomosis made after a partial gastrectomy for gastric ulcer carries a minimal risk of this eventuality.

After gastric resection for duodenal ulcer (and for stomal ulcer following gastro-jejunostomy) there is a very definite risk of stomal ulceration which appears to depend in part on the extent of the gastric resection. With the level of resection commonly carried out nowadays-in the region of a three-quarter resection-the writer finds a stomal ulceration recurrence rate of between 2 and 3 per cent. on a five year follow-up, and most cases which are going to relapse will do so within two to three years of the operation.

While partial gastrectomy must still be regarded as the orthodox operation for duodenal ulcer, there are other procedures to be considered.

Gastro-jejunostomy has but little place in the treatment of duodenal ulcer because of its high recurrent ulceration rate, between 30 and 40 par cent. if the cases are followed-up long enough. It is difficult to select special cases for short-circuiting on either the gastroscopic appearance of the mucosa or acidity levels, because these are variable and inaccurate methods of measuring the digestive powers. If the patient suffers from active duodenal ulceration it is likely that his digestive secretions are above average in quality and quantity, and any depression of acidity is likely to be transitory. The operation is justified in occasional intractable senile or fragile cases where an intervention is essential. It appears to be a satisfactory means of dealing with certain advanced cases of duodenal stenosis of the type seen in India and where opportunities for careful pre-operative preparation may be absent. It may be the most suitable operation for those surgeons in outlying posts who have had no opportunity of becoming practised in the operation of gastrectomy but who are compelled to deal with an occasional ulcer case.

Vagotomy as a solitary procedure must be and generally is abandoned because of its high incidence of persistent gastric stasis with the so-called side-effects of vagotomy, mainly foul belching and diarrhoea. In combination with partial gastrectomy, gastro-jejunostomy or pyloroplasty the operation is far more satisfactory and has many supporters. Although much has been written on the subject, no final judgment on its place in treatment can be given until there are many more reports on the progress of these patients between five and ten years post-operatively. Early reports, one to three years after operation are plentiful but 


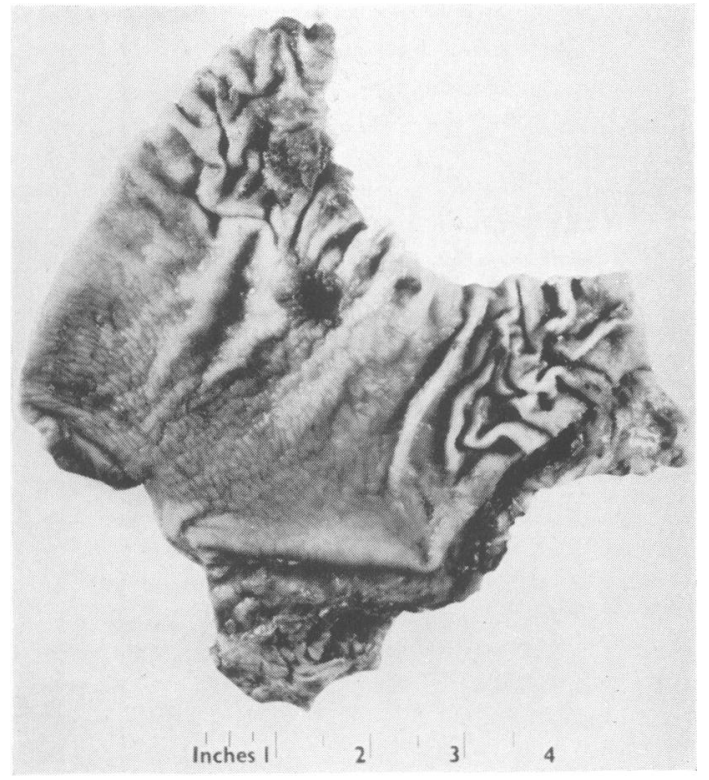

FIG. 2.- Gastric ulcer which was diagnosed for the first time and progressed two years after vagotomy for duodenal ulcer.

may be misleading in so far as the late ulcer prognosis is concerned. Some things we do know, however, from the shorter term reports.

We know that in up to 3 per cent. of cases gastric ulceration may progress and may even be diagnosed for the first time after vagotomy (Fig. 2). ${ }^{2}$ We know that if a vagotomy is combined with pyloroplasty, duodenal ulceration may persist or recur, or if it is combined with gastro-jejunostomy stomal ulceration may occur in at least 5 per cent. of cases. ${ }^{3}$ When combined with partial gastrectomy the incidence of stomal ulceration is low or zero, though the elapse of more time may cause opinions to change, particularly as the vagotomy is frequently combined with a purposely low resection. With regard to the latter combination the writer is of the opinion, bearing in mind that gastrectomy gives 97 per cent. freedom from stomal ulceration, that it is better to perform a reasonably high gastrectomy on all the duodenal ulcer cases and reserve vagotomy as a later procedure for the 3 per cent. of patients unlucky enough to develop anastomotic ulceration, rather than submit all the patients to the two operations for the sake of avoiding the discomfort of a second operation on 3 per cent. The combined procedure will no doubt carry a higher morbidity and mortality. ${ }^{4}$ If the resection is a low one it is
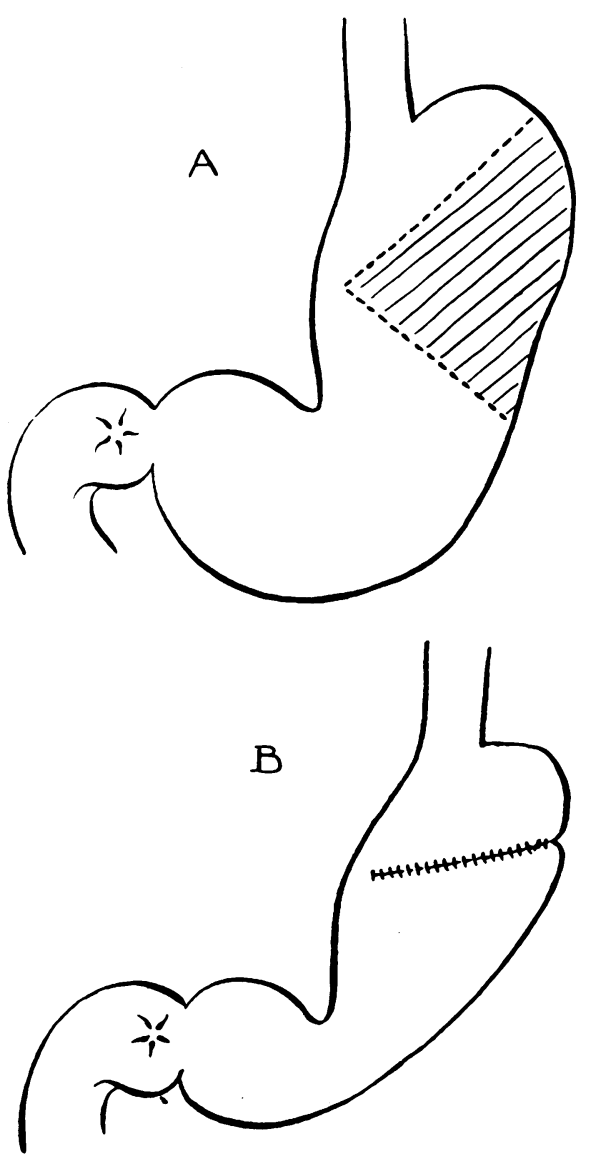

FIG. 3.- 'Fundusectomy' as suggested by Connell. This proved inadequate in the treatment of duodenal ulcer.

possible, though far from certain, that some of the post-gastrectomy symptoms may be avoided.

\section{Segmental Resection for Duodenal Ulcer}

This form of operation has been abandoned as inefficient in the past, but in its present more radical form it is possible that improved results may be obtained. In 1929 F. G. Connell ${ }^{5}$ described a method of treatment of duodenal ulcer in which he reduced the gastric acidity by resecting part of the fundus and greater curve of the stomach (Fig. 3). This was not very successful in reducing acidity, and $\mathrm{O}$. $\mathrm{H}$. Wangensteen tried variations in this operation by combining it with gastro-jejunal anastomosis or section of the anterior 0 vagus nerve, but without success. Later Wangensteen $^{6}$ greatly extended the operation by making a vast sleeve resection, removing all the body of the stomach from just below the cardia to the junction of body and the antrum. As this largely cuts the 


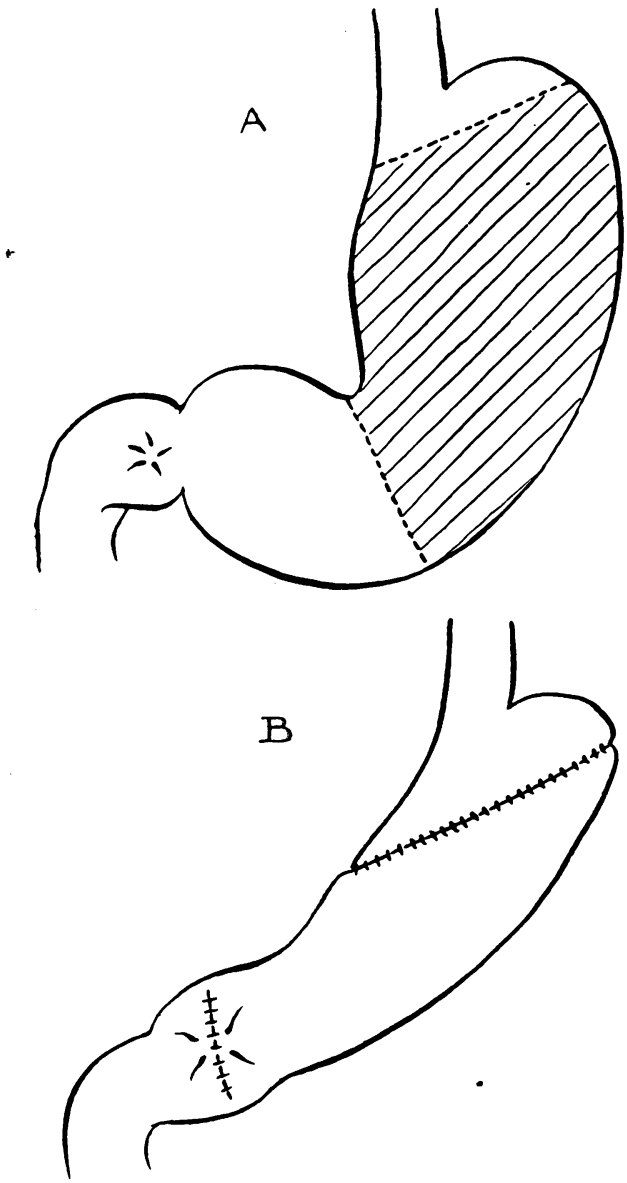

FIG. 4.-The 'Segmental Resection' advocated by Wangensteen. No long-term reports on results are available yet.

vagal fibres to the lower stomach, the risk of postvagotomy gastric stasis is overcome by the addition of a Heineke-Mikulicz pyloroplasty (Fig. 4). In order to approach the fundus adequately for the high gastro-gastric anastomosis, Wangensteen routinely splits the sternum up to the level of the fourth intercostal space. He claims that this form of resection gives a 65 per cent. incidence of achlorhydria to histamine injection and so it is possible that it will be more effective than the earlier operation against persistent duodenal ulcer, although there are as yet no long-term follow-up figures to confirm or refute this.

\section{Post Gastrectomy Symptoms and Other Un- toward Effects of Ulcer Operations}

Unfortunately a recital of the incidence of persistent duodenal ulcers or the development of new gastric or stomal ulcers does not exhaust the list of undesirable results in those who have undergone operations for peptic ulcer.

Post-gastrectomy symptoms. Perhaps too much has been made of the importance of these symptoms. While early post-cibal symptoms-a feeling of epigastric fullness with palpitations, sweating and perhaps fatigue or faintness are common in the early weeks after partial gastrectomy, they are slight in most cases and will not worry the patient unduly, particularly if the patient has been told to expect them and told that they will gradually diminish. If they arise without previous warning they may cause considerable alarm and undermine the patient's confidence. The extent to which they worry the patient usually varies inversely with the amount he has suffered from the ulcer. The patient who had little or no dyspepsia, e.g. if the gastrectomy was performed for massive haemorrhage from an acute ulcer, is more likely to be upset by such symptoms than the patient who has suffered for years with severe ulcer pain, though this is not invariably so.

It is well known too that some patients will exaggerate their symptoms, or add an excessive functional overlay to minor symptoms or find them a welcome excuse for delaying their return to an uncongenial job. Nevertheless it is undoubted that some patients with a good mental make-up and the desire to be well may be seriously disturbed by so-called dumping, and in a very small minority the symptoms may become even more disabling with time.

In some cases other symptoms may arise which may be unaccompanied but are usually associated with early dumping. Post-prandial looseness of the bowels is a troublesome complication. Loss of weight may occur more as a result of diminished intake of food than of failure to digest such food as it is taken (R. H. F. Brain). ${ }^{7}$ Biliary regurgitation is occasionally present and will lead to weight loss. Some patients lose their desire for certain foods-egg and milk are probably the two commonest articles. The cause for this antipathy is. uncertain. It has been suggested that a mild allergy to these foods may become evident after gastrectomy. However, other allergic symptoms are unusual and the patients usually state that these foods no longer attract them as they ' cause fermentation' in the stomach or cannot be digested.

What is the incidence of post-gastrectomy symptoms? The incidence depends on the length of time which has elapsed since operation, and recorded incidence varies with the care with which direct enquiry of the patient is made. Many factors come into play. If the patient suspects that an admission of symptoms will lead to further expense and time loss he may suppress information 
about them. Some patients are too grateful, because of their relief from pain, to mention or admit to these minor symptoms. However, with careful questioning the writer found that of patients who had had gastrectomies of the Polya type with a valve, performed for duodenal ulceration, some 7 per cent. had some degree of early dumping five years after operation. ${ }^{8}$ It is a fact that some surgeons claim to find no post-gastrectomy 'dumping' symptoms in their patients. Other observers who regard a feeling of epigastric ' fullness' after eating as a sign of dumping will find that over 90 per cent. of the patients have it at some time. These differences are of criteria of dumping rather than of incidence of dumping.

The reason why some patients are incapacitated by these symptoms is not known. They may appear after any form of gastrectomy. Similar symptoms are occasionally encountered in patients who have had no operation at all as a form of functional dyspepsia. Some have claimed that the incidence was less or negligible after the Billroth I form of operation. In a follow-up of cases operated on at St. James' Hospital the incidence was a little higher after the Billroth $I$ than the Polya, but it was significantly less persistent. In fact in the surgical treatment of severe and persistent post-gastrectomy symptoms, conversion of the gastro-jejunal into a gastro-duodenal anastomosis is the writer's most successful line of treatment. The interposition of loops of boweleither colon or jejunum-between stomach and duodenum is recommended by some writers, ${ }^{9,10}$ but these must be regarded as experimental and they have exceedingly risky possibilities. No very long-term follow-ups are available yet on these gastric ' replacement'. operations. Other operations used by the writer but far less satisfactory in small series, are short circuiting of the jejunal loops or conversion to a Roux form of anastomosis (both mainly employed for biliary regurgitation). As these latter procedures increase the liability to stomal ulceration the writer adds vagotomy for safety. Suspension of the stomach to avoid gravitational pull on the stomach and oesophagus is recommended by W. M. Capper and T. J. Butler. $^{11}$ Other operations found to have a varying measure of success are reduction of the size of the gastro-jejunal stoma, splanchnic injections, etc.

Such operations should not, of course, be carried out within a year of the original gastrectomy, for there is a tendency for these 'dumping' symptoms to diminish with time and they may lessen even as late as the third or fourth year.

Other troubles which may follow gastrectomy are as tollows.

Hypoglycaemic attacks. These are not com- mon but may lead to sweating with feeling of faintness and hunger arising one to two hours after a meal. They are best treated by avoiding starchy meals with much fluid (e.g. afternoon tea) and by maintaining a good protein and fat intake at each meal.

Iron deficiency anaemia. One cannot reduce the gastric acidity significantly without making the patient more susceptible to some of the disabilities which accompany a low gastric acidity. Diminished ability to absorb iron and perhaps vitamin $B$ may occur, so these articles should be taken in slight excess by the patient. In normal persons there is a tendency for the blood haemoglobin percentage to fall on the average as the years pass. In gastrectomized patients this fall is accelerated. Occasional patients develop severe secondary anaemia, but fortunately almost all of the cases respond very favourably to iron therapy.

There is a suspicion, as yet unproven, that a gastrectomy may increase the liability to biliary tract disease and to pulmonary tuberculosis, particularly if there has been much weight loss. These matters are being investigated.

Post-vagotomy symptoms. One of the earliest findings after vagotomy had become a common operation for duodenal ulcer was that some of the cases developed offensive sulphurous belching sometimes attended by abdominal cramps an diarrhoea. As these symptoms were associate with gastric stasis they were presumably due to ${ }^{+}$ abnormal protein break-down due to the combination of hypoacidity with gastric retention. Similar offensive belchings may occur in obstructive gastric cancer, but they do not occur in duodenal ulcer stenosis where the acidity is high. The condition became of negligible incidence when one of the factors, the stasis, was overcome by adding a routine 'drainage operation' to the vagotomy either gastro-jejunostomy, gastrectomy or pyloroplasty. Even diarrhoea is usually diminished or cured by relief of the gastric stasis. There is now no place for pure vagotomy in the treatment of duodenal ulceration (Fig. 5).

There are certain other troublesome 'side effects' of vagotomy. Cardiospasm may occur in a small number of cases but is readily and most simply relieved by passing a Hurst mercury bougie. The condition does not appear to persist.

Mild early morning looseness of the bowels is $N$ not uncommon but it does not disturb the patient unduly. Hypoglycaemia-like attacks may occur, O particularly during episodes of gastric stasis. It 0

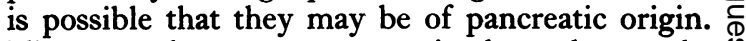
These attacks are commonest in the early months $\stackrel{\infty}{+}$ after operation.

Mortality of the operations. The operative mortalities and morbidities vary considerably from 

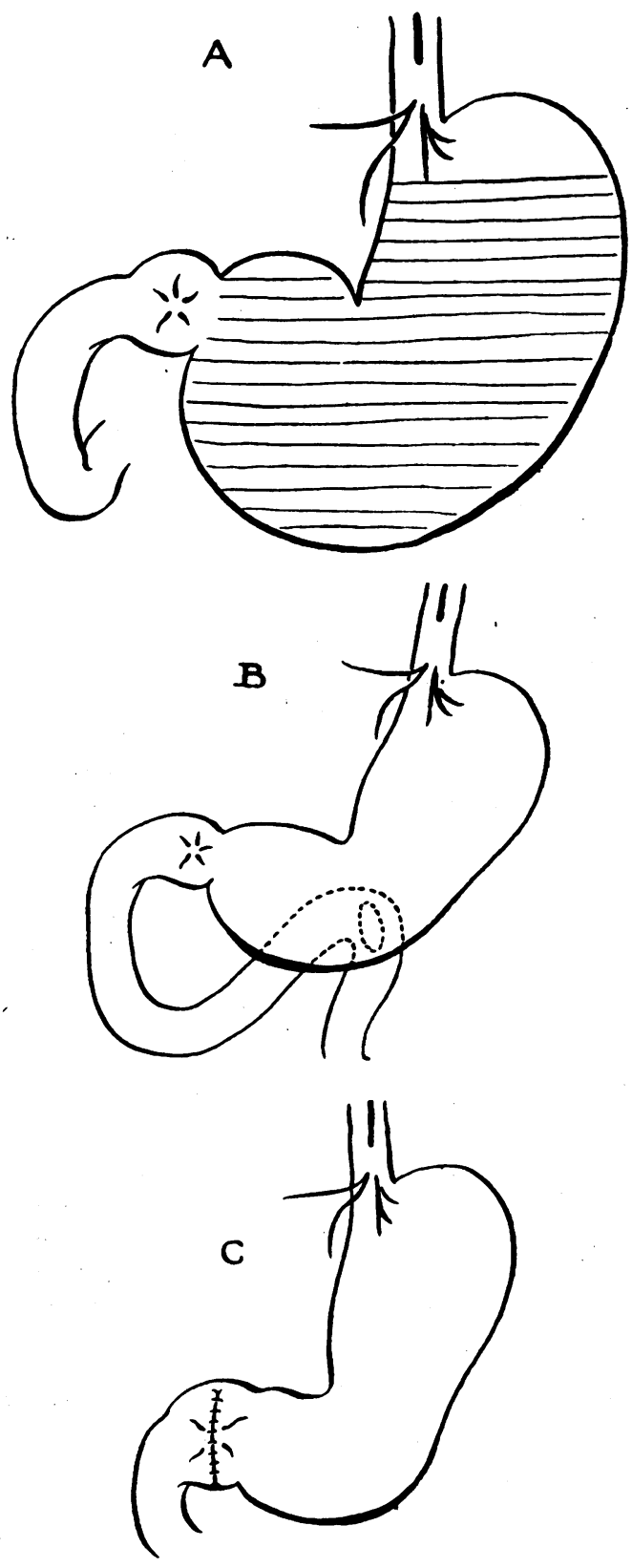

place to place. There is no doubt that although death occasionally follows vagotomy, sometimes due to generalized intestinal ileus or tachycardia, very rarely to oesophageal damage, its mortality is frequently under I per cent., whereas the mortality of gastrectomy is on the average reported at a slightly higher figure.

\section{Rationale of Treatment}

What, then, is the orthodox surgical treatment

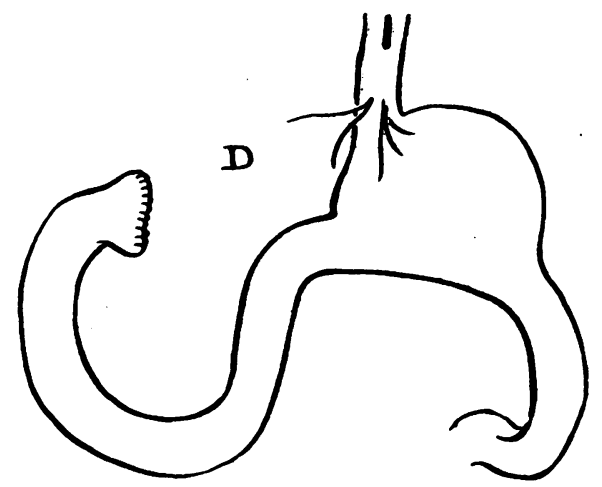

Fig. 5.- 'Drainage Operations.' Avoidance of the side effects of vagotomy by (b) gastro-jejunostomy, (c) pyloroplasty or (d) partial gastrectomy.

of gastric and duodenal ulcer ${ }^{8}$ ? For gastric ulcer a partial gastrectomy is required. As the duodenum is usually healthy in such cases, and in view of the fact that persistent 'dumping' is less common after the Billroth I (Péan) operation, a gastroduodenal reconstruction makes a very satisfactory conclusion to the gastrectomy. The operation is simpler, there are no jejunal loops to get into trouble and by mobilization of the second part of the duodenum, and by careful freeing of the greater curve of the stomach, a tension-free anastomosis "can always be made. As stomal ulceration is so uncommon after gastrectomy for gastric ulcer a particularly high gastrectomy need not be made. If the ulcer is high it may be possible to make a low gastric transection across the healthy greater curvature of the stomach, taking only a tongue-shaped upward extension of the lesser curve to include the ulcer and so avoid a high resection. If there is a high hour-glass deformity then a high gastric transection is unavoidable.

For duodenal ulceration there is no doubt that the orthodox operation is partial gastrectomy. One is often asked, "When should a vagotomy be done for duodenal ulceration ?' It is obvious that we cannot answer this question until we have a good series of reports on the state of the patient five to ten years after vagotomy. I believe, however, that it is justifiable under the following circumstances:

I. Where it is imperative to avoid weight loss as much as possible. I have used a combination of vagotomy and gastro-jejunostomy on patients with pulmonary tuberculosis who were suffering from an ulcer in which surgery was imperative, for example, in duodenal stenosis.

2. The young patient with a severe ulcer and high acidity. In several patients aged under 20 

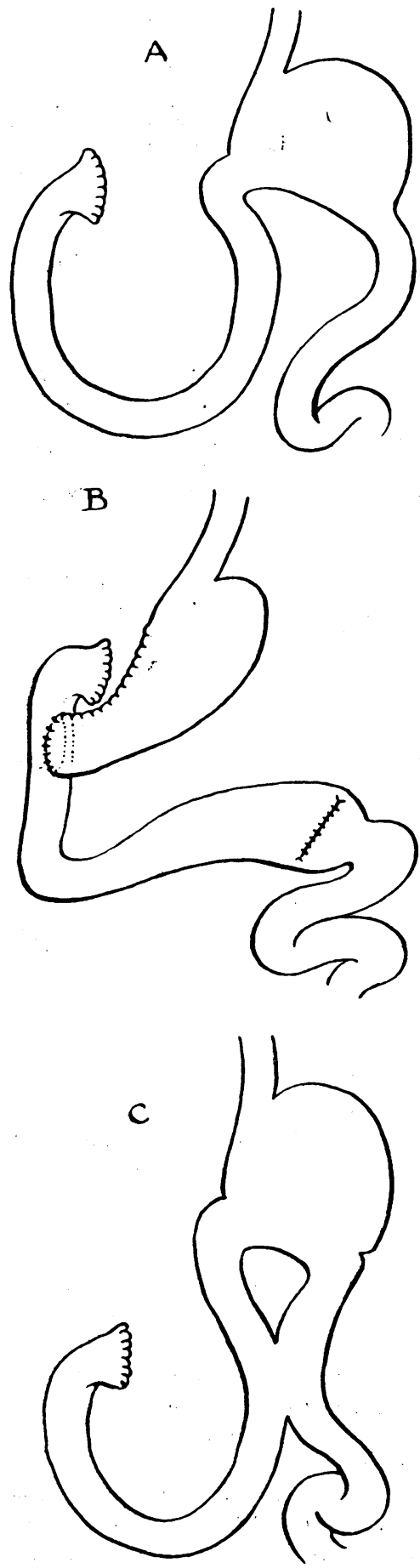

years I have carried out this operation believing that possibly there would be less interference with their statural development. The results have been

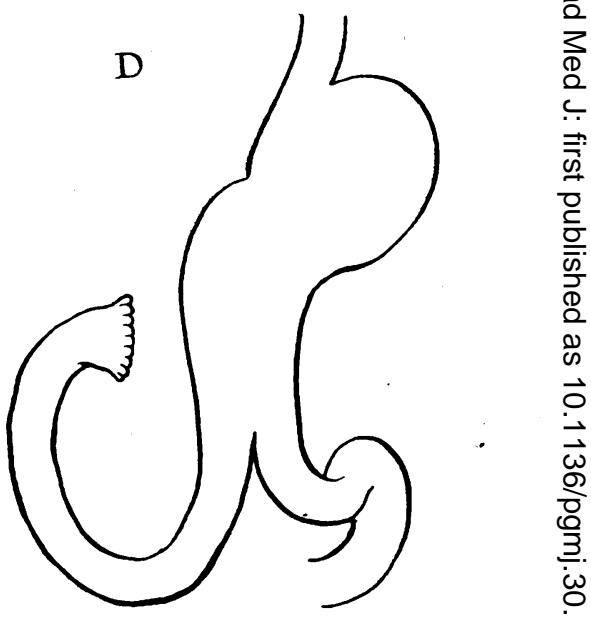

'FIG. 6.-Treatment of 'dumping' symptoms by (b) conversion to gastro-duodenal anastomosis, (c) short circuiting efferent and afferent jejunal loop $\overrightarrow{\hat{A}}$ and (d) the pantaloon operation.

very satisfactory, but on the other hand the few 20 to 30 year old patients on whom I have pero formed gastrectomy for intractable ulcer have alse made exceptionally good progress.

3. The patient with a long history, but whकें found at operation to have a minimal lesion. Such patients have obviously been much incapacitate $\Phi$ by a relatively minor lesion and as such a patien will be liable to make much of minor dumping symptoms, a vagotomy may be preferable.

4. The patient with an extensively penetrating duodenal ulcer which the operator feels would b $\overrightarrow{\vec{E}}$ hazardous to remove. In such a case a vagotomy and gastro-jejunostomy provides an alternative method of treatment. A gastrectomy with pre? pyloric transection is another approach to this problem, removing the antral mucosa at the same. time, or removing the whole antrum six week later as a second stage of the operation.

The correct selection of operation must awaie more complete information. In reports comparing vagotomy and partial gastrectomy and assessing ' satisfaction or dissatisfaction with the operation? the results seem roughly the same-about an. 85 per cent. satisfaction rate. ${ }^{12}$

If dumping proves troublesome after gastrec tomy, explanation, reassurance and medica management should first be tried, for example lying down for a short time after meals, small doses of barbiturate, dry meals of high proteit value and extra milk between meals. Furthe surgery should not be contemplated less than $\vec{a}$ year from the gastrectomy, but if the symptoms dg not abate, and particularly if there is much weight loss and biliary regurgitation, then reinterventiog 
is justifiable. A psychiatric opinion is often helpful, but it must be borne in mind that the lesion is never purely hysterical, though there may be a background or 'overlay' of neurosis.

If biliary regurgitation is the main complaint then reflux due to hiatus hernia or to obstruction of the stoma must be excluded. As indicated previously, the operation which the writer finds most satisfactory and which may even remove the troublesome idiosyncrasy to milk or eggs, is conversion from gastro-jejunal to a gastro-duodenal anastomosis (Fig. 6b). The writer has never found this impossible of performance, for the second and third parts of the duodenum can be extensively mobilized in order to anastomose the greater curve side of the stomach to the anterior surface of the second part of the duodenum. If the stomach is extensively adherent however, as, for example, after radical gastrectomy for cancer, then it may be simpler to try a long anastomosis of the afferent and efferent loops, the so-called 'pantaloon operation' (Fig. 6d) which is moderately effective.

\section{REFERENCES}

I. TANNER, N.C. (1951), Edin. Med. F., 58, 26r .

2. JOHNSON, H. D., and ORR, I. M. (1953), Lancet, i, 253

3. CRILEX, G., Jun. (1952), Ann. Surg., 136, 752.

4. DRUCKERMAN, L. J. WEINSTEIN, V. A KLINGENSTEIN, P., and CÖLP, R. (1953), 'f. Amer. Med. Ass., I51, 1266.

5. CONNELL, F. G. (1929), Surg. Gynec. and Obstet., 49, 696.

6. WANGENSTEEN, O. H. (1952), 'F. Amer. Med. Ass., 149, 18.

7. BRAIN, R. H. F. (1953), Proc. Roy. Soc. Med., 46, 438.

8. TANNER, N. C. (1948), W. Lond. Med. F., 53, 35.

9. HENLEY, F. A. (1952), Brit. F. Surg., 40, 118.

ro. MORONEY, J. (195 I), Lancet, i, 993.

I I. CAPPER, W. M., and BUTLER, T. J. (195I), Brit. med. f. ii, 265 .

12. POLlaRD, H. M., BOLT, R. J., RANSOM, H. K., and

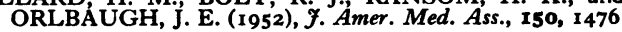

\title{
THE PATHOLOGY OF PEPTIC ULCERATION
}

\author{
By H. A. Magnus, M.D., M.R.C.P. \\ Professor of Morbid Anatomy, University of London, Director of Pathology Department, King's College Hospital
}

In this paper the remarks have been confined to the consideration of the pathology of chronic gastric and duodenal ulceration and the problem of ulcer-cancer. Whilst it is true that, at its conception, every chronic ulcer must have passed through the stage of erosion and acute ulceration, it seems unlikely that such lesions, when multiple and part of an acute erosive gastritis, commonly precede the development of one or more chronic ulcers.

The figures given in this paper are based on an analysis of 944 partial gastrectomy specimens removed for peptic ulceration. In comparing these figures with those of previous workers it must be remembered that gastric resection is now one of the commonest operations performed by the general surgeon, whilst at the time when most of the authoritative papers on this subject were written it was usually only undertaken as a last resort.

Of the 944 specimens examined, 523 were removed for duodenal ulceration and 421 for gastric ulceration. In eight of the specimens there was evidence of both duodenal and gastric ulceration. In three of these both the gastric and duodenal ulcers were active, in two the gastric ulcers were active and the duodenal represented by puckered scars and in three the duodenal ulcers were active and the gastric represented by healed puckered scars. Thus 1.5 per cent. of chronic duodenal and 2.5 per cent. of chronic gastric ulcers were accompanied by evidence of chronic ulceration of the other organ. These figures are considerably lower than Stewart's (Hurst and Stewart, 1929), but his figures were based on an analysis of 4,000 autopsies and showed that 9 per cent. of chronic duodenal and 12 per cent. of chronic gastric ulcers were accompanied by chronic lesions of the other organ. These figures are much more likely to give a true figure of the frequency of association of lesions in the two organs. Whilst in surgical material some duodenum is frequently included, this is not always the case and some duodenal lesions are bound to be missed.

\section{Chronic Gastric Ulcer}

Sex Incidence and Age

Of the 421 gastrectomy specimens showing chronic gastric ulcers, 322 were from males and 99 from females, giving a ratio of $3.2: 1$. This is a very different figure from that given by Hurst and 